\title{
Iterative nonlinear joint transform correlation for the detection of objects in cluttered scenes
}

\author{
T. Haist and H.J. Tiziani \\ Institut für Technische Optik, Universität Stuttgart
}

\begin{abstract}
An iterative correlation technique with digital image processing in the feedback loop for the detection of small objects in cluttered scenes is proposed. A scanning aperture is combined with the method in order to improve the immunity against noise and clutter. Multiple reference objects or different views of one object are processed in parallel. We demonstrate the method by detecting a noisy and distorted face in a crowd with a nonlinear joint transform correlator.
\end{abstract}

Index Terms-image processing, object registration, object detection, correlation, joint transform correlator, feedback, iterative methods, face recognition, pattern recognition

\section{INTRODUCTION}

$\mathbf{C}$ ORRELATION methods are well established techniques for object positioning and object recognition[1]. Digital electronics[2] and different optical setups[1], [3], [4], [5] have been used to compute the cross-correlation between image and reference patterns. Among the optical methods, nonlinear joint transform correlators (JTC) are often employed because of the ease of implementation. Furthermore, the reference patterns are directly useable at the input stage and no filter generation is necessary. Therefore, real-time operation is possible.

Optimized filters[6], [7], [8], [9], [10], the incorporation of three-dimensional information[11], [12], multiple input images[13], [14], [15], pre- and post-processing[16], [17], [18], and different nonlinear techniques[19], [20], [21], [22] are suitable methods to improve the correlator performance. In this article, we concentrate on an iterative correlation technique for the detection of small objects in cluttered scenes (non-overlapping noise).

The basic idea of iterative correlation goes back to work of Smolińska[23], Iwaki et al[24], Alam and Karim[25], [26], and $\mathrm{Yu}$ and Cheng[27]. Edwards and Murase[28] used a correlation based approach in the spatial domain in order to detect multiple copies of one reference object. Smolińska[23] employed a scanning aperture for the correlation of small parts of a complex scene with simple patterns like circles. An electronic feedback system guided the aperture of the Van der Lugt correlator to the regions of main interest within the scene. Iwaki et al[24], [29] and Alam and Karim[25] improved a bipolar JTC by arranging the input and reference objects in a circular arrangement. First, the cross-correlation was computed. After that, the different input patterns were multiplied with the corresponding correlation peak intensities and a new input image was generated. Alam and Karim also proposed to apply additive instead of multiplicative feedback[26]. Yu and Cheng[27] used a similar approach where one input object and multiple reference objects were presented simultaneously. For their experiments, the authors used alphabetic characters.

The disadvantage of these methods is the need to know the positions of the input patterns in advance. We circumvent this problem by using digital image processing within the feedback loop of the correlator. By this approach, we obtain a significantly improved correlator output for very complicated scenes even if multiple reference patterns have to be detected. The sensitivity against noise is further reduced by a scanning aperture.

We demonstrate the feasibility of the method by detecting a small face in a crowd with a simulated nonlinear JTC. Although the face is blurred, noisy, sub-sampled, and has a nonlinear transformed histogram, scale-invariant detection is possible.

\section{ANALYSIS}

In Fig. 1 a complex scene including different faces is shown. We want to detect one specific face (reference). The reference face is very small compared to the whole image (relative area $<1 \%$ ) and a lot of similar faces are present. To make the problem more difficult, we smoothed the face in the input image, transformed the histogram by $I^{\prime}=I^{1.5}$, and subsampled the whole image. Magnified versions of the face to be detected and the reference pattern are shown in Fig. 2.

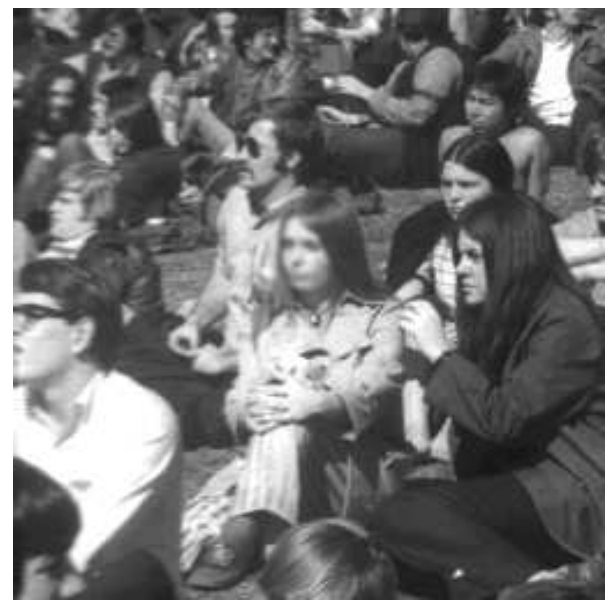

Fig. 1. input scene

Chellappa et al[30] gave a good introduction to ordinary digital image processing approaches to the problem of face recognition. Javidi et al used a nonlinear joint transform 


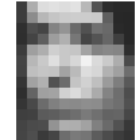

(a) reference pattern

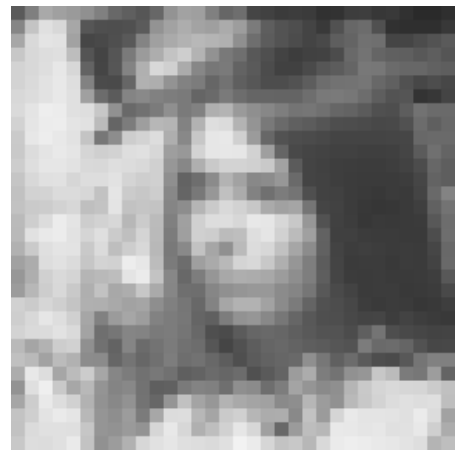

(b) corresponding area within the scene in Fig 1.
Fig. 2. reference pattern and modified part of the input scene

correlator for the implementation of a neural network in order to achieve robust face recognition with multiple reference faces[31]. Their system yields very good discrimination among different faces although it is based on a non-iterative technique and therefore faster that the method we propose in the rest of the this paper. However the motivation of this work differs from ours in that we want to detect small faces in very cluttered scenes. Both methods may be combined in order to first detect a face with our system and then to use the approach in Ref. [31] to further evaluate the detected face without the cluttered background.

Apart from correlation methods, considered here, morphological processing[32], [33] seems to be especially convenient for the detection of small objects in cluttered scenes.

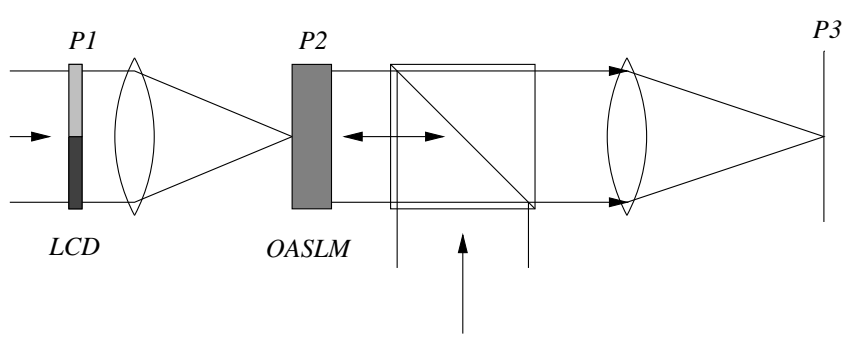

Fig. 3. optical JTC implementation

In the following, we shortly summarize the basic principle of joint transform correlation. Fig. 3 shows one typical optical implementation of nonlinear joint transform correlation. The input image $O(x, y)$ (input) and the reference object $R(x, y)$ (reference) are written side by side into one spatial light modulator. A lens performs the Fourier transform. The intensity of the Fourier transform, for example generated by an optically addressed light modulator[34], [35], is called the joint transform power spectrum (JTPS). The correlation is obtained by Fourier transforming this JTPS back into the spatial domain.

If the input and the reference are separated by $2 x_{0}$, then the complex light field at the plane $P 1$ (Fig. 3) is

$$
I(x, y)=O\left(x-x_{0}, y\right)+R\left(x+x_{0}, y\right) .
$$

The JTPS then is given by

$$
\begin{aligned}
j\left(f_{x}, f_{y}\right)= & \left|r\left(f_{x}, f_{y}\right)\right|^{2}+\left|o\left(f_{x}, f_{y}\right)\right|^{2} \\
& +r^{*}\left(f_{x}, f_{y}\right) o\left(f_{x}, f_{y}\right)+r\left(f_{x}, f_{y}\right) o^{*}\left(f_{x}, f_{y}\right),
\end{aligned}
$$

where we used the reduced coordinates $f_{x}=x_{2} /(\lambda f)$ and $f_{y}=y_{2} /(\lambda f)$ with the focal length $f$ of the Fourier transforming lens. $\lambda$ denotes the wavelength and $\left(x_{2}, y_{2}\right)$ are the spatial coordinates in the Fourier plane P2. $r$ and $o$ are the Fourier transforms of $R$ and $O$.

The inverse Fourier transform of Eq. 3 yields

$$
\begin{aligned}
C(x, y)= & R(x, y) \otimes R(x, y)+O(x, y) \otimes O(x, y) \\
& +O\left(x+2 x_{0}, y\right) \otimes R(x, y) \\
& +O\left(x-2 x_{0}, y\right) \otimes R(x, y),
\end{aligned}
$$

where $\otimes$ denotes correlation. The first two terms of Eq. 3 are the auto-correlations, the third and the fourth term are the cross-correlations.

Improvement of the correlation peak quality is possible by the introduction of nonlinear transforms to Eq. 3 before the inverse Fourier transform is performed. Well known techniques are binarization[36], [21], [20], [22], k-th law nonlinearities[37], [38], and windowing[36]. For our simulations we used an easily implementable (for example by optically addressed liquid crystal displays[34], [35]) k-th law nonlinearity with $k=0.5$, that is the JTPS $j$ is transformed by

$$
j^{\prime}=\sqrt{j}
$$

prior to the inverse Fourier transform. All simulations were done with additional zero-padding in the input plane to avoid aliasing due to the discrete Fourier transform (circular correlation error).

\section{ITERATIVE CORRELATION}

Although nonlinear joint transform correlation is a powerful method for object recognition, very demanding problems like the face in the crowd lead to a poor overall performance of the correlator. In Fig. 4, the intensity in the correlation plane for the correlation between the scene (Fig. 1) and the reference face (Fig. 2) is shown. This correlation result is not satisfactory because strong noise hides the correlation peak. We now explain an iterative correlation technique that significantly improves the correlator output.

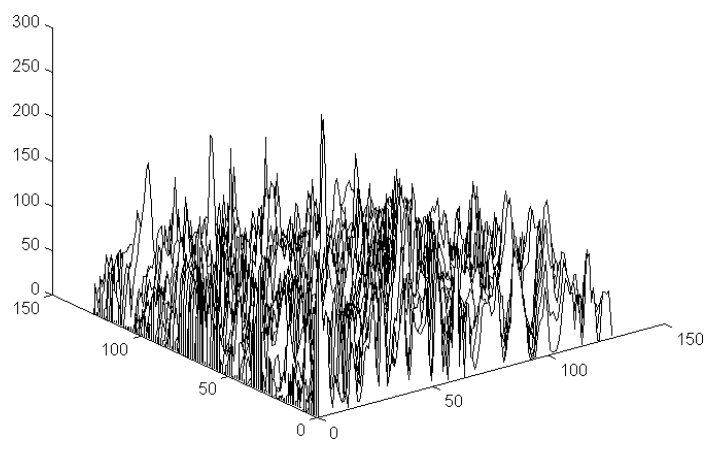

Fig. 4. nonlinear JTC correlation of Fig. 1 with Fig. 2 (a) 


\section{A. Single reference case}

The basic idea of the proposed method is to use the correlation result of Fig. 4 to modify the input image. The correlator output $C(x, y)$ is used to compute a mask that is multiplied with the original input image to obtain a new input image for the JTC. The algorithm for the mask computation is depicted in Fig. 5: First, the mask is initialized with a fixed constant $c_{2}$. Then, $C(x, y)$ is normalized to 255 and lexicographically scanned. If a peak value $C\left(x_{p}, y_{p}\right)$ exceeds a fixed threshold, the peak at this position $\left(x_{p}, y_{p}\right)$ is further evaluated: we scan the mask over the reference pattern area at the position in the mask that corresponds to the peak position. At each position in the mask, the new value $C\left(x_{p}, y_{p}\right)^{c 1}$ is written into the mask if $C\left(x_{p}, y_{p}\right)^{c 1}$ exceeds the old mask value. $c_{1}$ is constant (see below). In other words: we replace the mask with the maximum of the old mask and a rectangle with the size of the reference pattern at the position of the peak and with the value $C\left(x_{p}, y_{p}\right)^{c 1}$. A typical mask is shown in Fig. 6.
7. The peak that corresponds to the reference face now is easily detectable, and the position of the object is correctly determined. Some noise and broadening of the peak still remains due to differences between the reference and the input image. Therefore, the peak position - and the object position - can only be determined within an accuracy of two or three pixels. The important point here is that the cluttered background has been reduced and no false peaks have been detected. Additional digital post-processing by smoothing can be used to improve the output quality of the correlator.

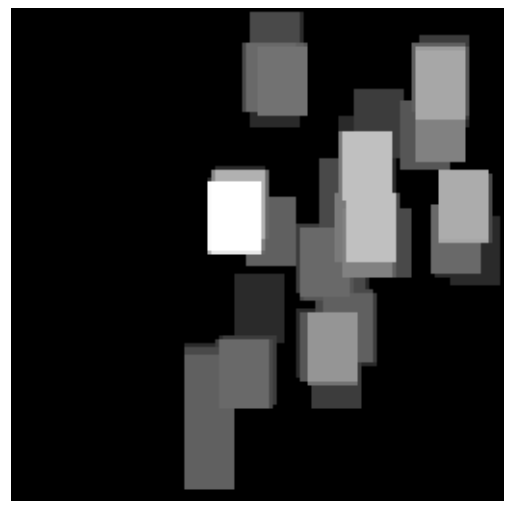

Fig. 6. mask computed with the algorithm in Fig. 5

Within the algorithm, three constants $c_{1}, c_{2}$, and $c_{3}$ are used. $c_{2}$ is used to initialize the mask and is therefore the mask background. $c_{1}$ is approximately 1 and can be used to further amplify strong peaks. A large value for $c_{1}$ therefore speeds up the iteration whereas a large value of $c_{2}$ tends to lower the convergence rate. Only peaks of $C(x, y)$ greater than $c_{3}$ are evaluated. Therefore it is necessary that $c_{3}$ is chosen to be less than the minimum correlation value that one obtains for the object to be detected. Large values tend to improve the convergence speed. We set this parameter to 70 throughout our experiments, but it may be necessary to change this value for other applications. For very large images with small target objects it might be necessary to reduce $c_{3}$. For large target objects one might try to increase $c_{3}$.

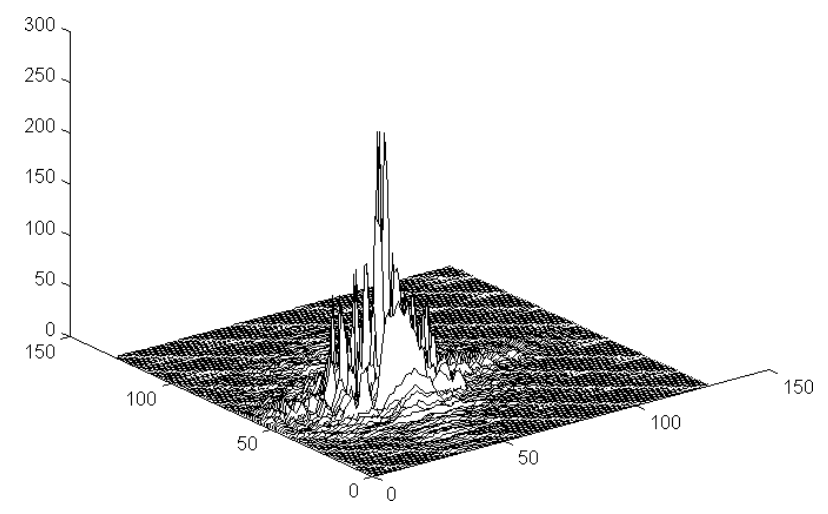

Fig. 7. nonlinear JTC correlation of Fig. 1 with Fig. 2 (a) with 3 iterations

In order to obtain suitable values for $c_{1}$ and $c_{2}$, we ran the whole simulation with different values for $c_{1}$ and $c_{2}$. The until a predetermined number of iterations is reached. After three iterations, we obtain the correlation result shown in Fig.
Fig. 5. mask computation within feedback (single reference case)

The new input image is obtained by the multiplication of this mask with the last input image. Again, the correlation is performed, and we iteratively repeat the whole process

,

.


results are presented in Table I. The tabulated values show the peak-to-average-correlation energy $(P C E)$, defined as the ratio of the correlation peak and the total energy in the correlation plane, and the number of iterations necessary to obtain a $P C E>0.003$. Empty boxes $\left(c_{2} \geq 1.3\right.$ or $\left.c_{2} \leq 0.8\right)$ indicate that the correct solution wasn't found for the choice of parameters. For the problem at hand, $c_{1}=1.1$ and $c_{2}=30$ seems to be a good choice. These values were therefore used for the rest of our experiments.

As it is well known, the nonlinearity employed in the Fourier domain often significantly affects the discrimination ability. Here, we observed a rather weak influence probably due to the small reference pattern area. The performance of the system began to decrease significantly (approximately by 20 per cent) if small $(k=0.8)$ or large $(k=0.2) \mathrm{k}$-th law nonlinearities were used. Detailed investigations concerning the optimum nonlinearity are an option for future research. Here we concentrated on the iterative method and used only the $k=0.5$ nonlinearity for the experiments.

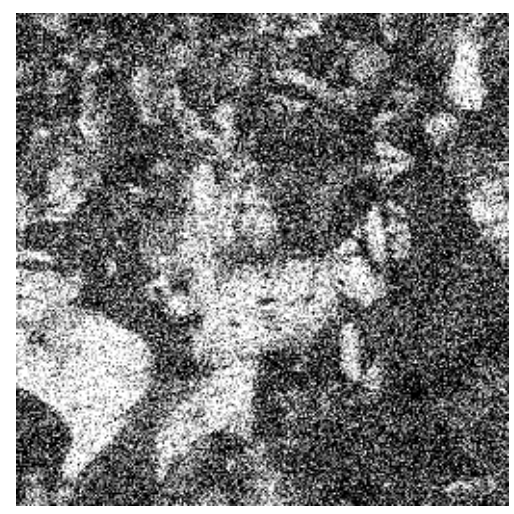

Fig. 8. input scene of Fig. 1 with additive Gaussian noise $(\sigma=0.3)$

We added different amounts of Gaussian noise to the already smoothed input image to test the resistance against additive noise. No smoothing was used after the noise had been added. Even with quite strong noise (standard deviation $\sigma=0.17$ ), face recognition is possible. The final PCE (after iteration) is constant (within statistical limits). If very noisy images $(\sigma \geq$ 0.2 ) are used, the iteration converges to the wrong solution. A simple approach to improve the JTC performance for small references is to divide the input image into smaller sub-images that are correlated one after the other with the reference pattern[23]. For every single correlation, the percental amount of clutter is therefore strongly reduced. By dividing the input plane into $4 \times 4$ sub-images (with an overlap of $50 \%$ ) we can detect the reference face even with strong noise $(\sigma=0.4)$. An image distorted by $\sigma=0.3$ additive noise is shown in Fig. 8 .

The computational cost of the aperture approach is unfortunately increased compared to the conventional crosscorrelation. For the correlation of two $256 \times 256$ images the number of multiply-add computations (MAC) is[2] $545 \cdot 10^{6}$ whereas for a $512 \times 512$ image $4.9 \cdot 10^{9}$ MAC are necessary. Due to the necessary overlap between the sub-images (we have to be sure that the pattern we are looking for is not cut into two halves) $4^{2}=16$ correlations are necessary. Therefore, the overall computational cost is increased by a factor of $16 * 545 \cdot 10^{6} / 4.9 \cdot 10^{9}=1.8$.

This number departs (due to real world effects) from the theoretical factor of about four, which one obtains by using the simple proportionality 'computational cost per $1 \mathrm{D}$ FFT $\sim$ $\mathrm{n} \log _{2}$ n'.

\section{B. Multiple reference case}

In the preceding section, a single reference pattern has been recognized. Now, we explain how some additional image processing within the mask computation is employed in order to search for different reference patterns.

The main problem with multiple reference patterns is that it is not clear to which reference pattern a correlation peak corresponds. Take as a simple example two reference patterns that are presented simultaneously to the correlator: because the reference patterns are spatially separated, a peak could correspond to position 1 in the input plane and pattern 1 or position 2 and pattern 2. Therefore, it is in general not clear which parts of the input image are to be amplified by the mask.

The basic approach of correlation can be visualized in the spatial domain as sliding the reference pattern across the input image. For every position of the sliding window, the sum of the multiplications of the reference pixels with the pixels of the input image is computed. Another approach, quite similar to correlation, is to add the absolute differences between the reference pixels and the pixels of the input image. Obviously this sum of the absolute errors $(S A E)$ is minimal if the sliding window is at the correct position, corresponding to the position of the reference face in the scene. Mathematically

$$
S A E(i, j)=\sum_{x, y \in \Lambda}|O(i+x, j+y)-R(x, y)|,
$$

where $\Lambda$ denotes the reference pattern area.

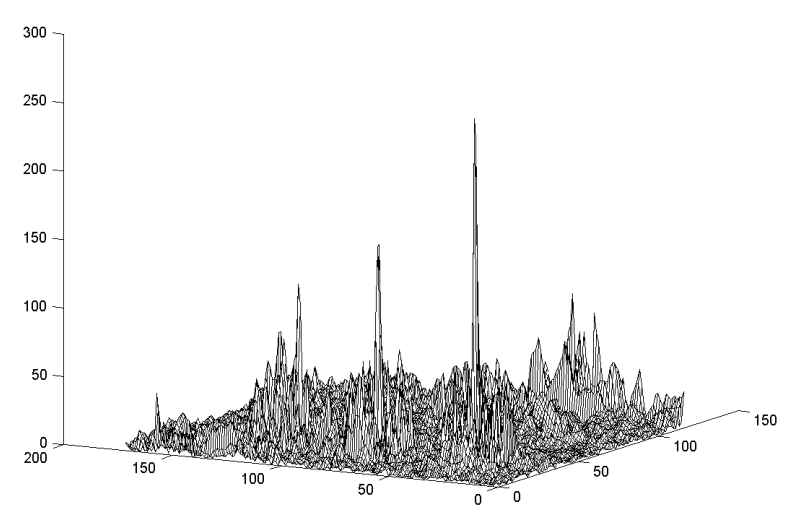

Fig. 9. Nonlinear JTC correlation with three reference patterns: result for subarea of the noisy $(\sigma=0.2)$ input imag. The strong peak correspondes to the correct reference pattern.

If the pattern in the scene corresponds exactly (same digital gray-levels) to the reference pattern, the $S A E$ is zero at 


\begin{tabular}{|c|c|r|r|r|r|r|r|}
\hline$c_{2}$ & & $c_{1}=0.8$ & $c_{1}=0.9$ & $c_{1}=1.0$ & $c_{1}=1.1$ & $c_{1}=1.2$ & $c_{1}=1.3$ \\
\hline \hline 10 & $\mathrm{PCE} * 10^{-3}$ & & 4.4 & 5.9 & 4.9 & 4.0 & \\
& iterations & & 3 & 3 & 2 & 4 & \\
\hline 20 & $\mathrm{PCE} * 10^{-3}$ & & 4.8 & 3.1 & 4.9 & 5.9 & \\
& iterations & & 3 & 2 & 2 & 3 & \\
\hline 30 & $\mathrm{PCE} * 10^{-3}$ & & 4.3 & 3.1 & 5.4 & 3.9 & \\
& iterations & & 3 & 2 & 2 & 4 & \\
\hline 40 & $\mathrm{PCE} * 10^{-3}$ & & 3.2 & 5.6 & 5.1 & 4.5 & \\
& iterations & & 3 & 3 & 2 & 3 & \\
\hline 50 & $\mathrm{PCE} * 10^{-3}$ & & 4.1 & 5.3 & 4.7 & 4.0 & \\
& iterations & & 4 & 3 & 2 & 3 & \\
\hline 60 & $\mathrm{PCE} * 10^{-3}$ & & 4.1 & 4.9 & 4.1 & 5.1 & \\
& iterations & & 5 & 3 & 2 & 3 & \\
\hline 70 & $\mathrm{PCE} * 10^{-3}$ & & 3.9 & 4.2 & 3.6 & 5.1 & \\
& iterations & & 6 & 3 & 2 & 3 & \\
\hline 80 & $\mathrm{PCE} * 10^{-3}$ & & 3.0 & 3.6 & 3.1 & 5.8 & \\
& iterations & & 7 & 3 & 2 & 3 & \\
\hline 90 & $\mathrm{PCE} * 10^{-3}$ & & & 4.8 & 3.0 & 5.4 & \\
& iterations & & & 4 & 4 & 3 & \\
\hline
\end{tabular}

TABLE I

PCE AND NUMBER OF NECESSARY ITERATIONS FOR $P C E>0.003$ FOR DIFFERENT PARAMETERS $c_{1}$ AND $c_{2}$

the correct position. Due to distortions, noise, and different histograms this is unfortunately not the case for practical applications like the problem at hand. Here, direct evaluation of the $S A E$ is not suitable because the signal to noise ratio is very low.

Nevertheless, the $S A E$ is a valuable parameter because it is low if the patterns are similar and high if they are different. Therefore, we employ the $S A E$ within the mask computation for multiple reference patterns. The following strategy is used: after a reasonably high peak has been found in the correlation plane (see Fig. 6), the $S A E$ is computed for all the possible positions (corresponding to the peak) in the input scene using the corresponding reference patterns. Only the part of the input scene that corresponds to the minimal $S A E$ is amplified.

For large reference patterns, the $S A E$ computation can be a time consuming process if the whole image is processed. Acceleration is possible by going directly to the next position of the sliding window as soon as it becomes obvious that a position is not correct or at least probably not correct[39].

Combined with iterative correlation, only a few positions of the sliding window have to be examined and the reference patterns are small (for example $20 \times 30$ pixels). Therefore, the computational cost is negligible, as compared to the Fourier transforms.

We tested the $S A E$ approach by using three reference patterns. The three different sized versions of the reference face were applied in parallel by arranging them at different positions in the input plane in order to obtain a scale-invariant detection of the face. We modified the input image by copying a magnified (magnification $=2$ ) version of the reference face into it. Both faces, now present in the input image, were correctly detected even at high noise levels $(\sigma=0.2)$. The peak corresponding to the small face is shown in Fig. 9 together with the smaller peaks due to the other reference pattern. As described above, the SAE is used in order to decide which one of the peaks is correct. For the subarea with the large face, a similar correlation result is obtained. We can also use rotated faces or multiple faces instead of scaled versions of one reference face.

Moreover, we employ the $S A E$ as an additional parameter in order to decide if the result obtained with the iterative correlation method really corresponds to a reference pattern. This is necessary because iterative correlation converges to a (wrong) solution with low PCE and high $S A E$ if no reference pattern is present in the input scene. For our example, it was always sufficient to use the condition normalized $P C E>$ 0.001 AND $S A E<5 \cdot 10^{-12}$ as a criterion to decide if the obtained solution corresponds to a reference pattern.

Other approaches for scale-invariant detection were used in the past[40], [41], [42]. We tried to use the Fourier-Mellin[40], [43], [44], [45] transform within the iteration but failed to obtain acceptable results probably due to the complexity of the input scene. The basic idea to correlate the Fourier power spectra (which are shift invariant and therefore tractable by the Mellin transform) is not suitable here because the information of the spatial position of spatial frequencies within the images is lost due to the elimination of the phase of the Fourier transform. But this information is important if we want to detect a face within a scene that contains other faces because the characteristic spatial frequencies are very similar, and therefore additional information about the position of these frequencies is necessary.

\section{CONCLUSIONS}

We have presented an iterative correlation method that employs digital image processing within the feedback loop. By this approach, the performance of a simulated nonlinear joint transform correlator has been improved in order to detect distorted and noisy objects in cluttered scenes. The iterative correlator was able to detect small patterns that were not detectable with the one-pass correlator. We have demonstrated detection with multiple reference patterns in parallel, and a scanning aperture at the input plane has been used to improve the immunity against Gaussian noise and clutter.

We thank the Deutsche Forschungsgemeinschaft (DFG) for financial support. 


\section{REFERENCES}

[1] F. T. S. Yu and D. A. Gregory, "Optical pattern recognition architectures and techniques", Proceedings of the IEEE Vol. 84, pp. 733-752 (1996).

[2] M. Cavadini, M. Wosnitza, M. Thaler, and G. Tröster, "A VLSI architecture for real time object detection on high resolution images", Proc. of European Signal Processing Conference (EUSIPCO-96) Vol. 3, pp. 1475-78 (1996).

[3] J. W. Goodman, "Introduction to Fourier optics", McGraw Hill, 2nd Edition (1996).

[4] A. W. Lohmann, "Matched filtering with self-luminous objects", Applied Optics 7, pp. 561-563 (1968).

[5] Q. Tang and B. Javidi, "Multiple object detection with a chirp-encoded joint transform correlator", Applied Optics 32, pp. 5079-5088 (1993)

[6] F. Wyrowski, "Digital phase-encoded inverse filter for optical pattern recognition", Applied Optics 30 , pp. 4650-4657 (1991).

[7] J. Rosen and J. Shamir, "Application of the projection-onto-constraintsets algorithm for optical pattern recognition", Optics Letters 16, pp. $752-754$ (1991).

[8] B. V. K. V. Kumar, "Tutorial survey of composite filter designes for optical correlators", Applied Optics 31 , pp. 4773-4801 (1992).

[9] F. T. S. Yu, M. Lu, G. Lu, S. Yin, T. D. Hudson, and D. K. McMillen, "Optimum target detection using a spatial-domain bipolar composite filter with a joint transform correlator", Optical Engineering 34, pp. 3200-3206 (1995).

[10] U. Mahlab, J. Rosen, and J. Shamir, "Iterative generation of complex reference functions in a joint-transform correlator", Optics Letters 16 , pp. 330-332 (1991).

[11] T. Haist and H.-J. Tiziani, "Fast positioning of non-cooperative objects", Proceedings of the SPIE Vol. 3098, pp. 424-430 (1997).

[12] J. Rosen, "Three-dimensional electro-optical correlation", J. Opt. Soc. Am. A 15, pp. 430-436 (1998).

[13] M. Schönleber, H. Cedilnik, and H.-J. Tiziani, "Joint transform correlator subtracting a modified Fourier spectrum”, Applied Optics 34, pp. 75327537 (1995)

[14] X. Huanf, H. Lai and Z. Gao, "Multiple-target detection with use of a modified amplitude-modulated joint transform correlator", Applied Optics 36 , pp. 9198-9204 (1997).

[15] H. Su and M. A. Karim, "Performance improvement of a phase-shifting joint transform correlator by use of phase-iterative technique", Applied Optics 37 , pp. 3639-3642 (1998).

[16] R. R. Kallman and D. H. Goldstein, "Phase-encoding input images for optical pattern recognition", Optical Engineering 33 , pp. 1806-1812 (1994).

[17] T. Wolf, B. Gutmann, H. Weber, J. Ferré-Borrull, S. Bosch, and S. Vallmitjana, "Application of fuzzy-rule-based postprocessing to correlation methods in pattern recognition", Applied Optics 35 , pp. 6955-6963 (1996).

[18] Y. R. Wang, L. Z. Cai, H. Wang, and H. Liu, "High-efficiency hybrid joint-transform correlator with pre-processed input image and replicated binary spectrum", Journal of Modern Optics 44, pp. 1197-1205 (1997).

[19] T. Grycewicz and B. Javidi, "Experimental comparison of binary joint transform correlators used for fingerprint identification", Optical Engineering 35 , pp. 2519-2525 (1996).

[20] J. Wang and B. Javidi, "Multiobject detection using the binary joint transform correlator with different types of thresholding methods", Optical Engineering 33 , pp. 1793-1804 (1994).

[21] W. B. Hahn and D. L. Flannery, "Design elements of binary joint transform correlation and selected optimization techniques", Optical Engineering 31 , pp. 896-905 (1992).

[22] B. Javidi and C.J. Kuo: "Joint transform image correlation using a spatial light modulator at the Fourier plane", Applied Optics 27, pp. 663-665 (1988).

[23] B. Smolińska, "Scene analysis implemented optically", Optik 81, 163166 (1988).

[24] T. Iwaki, Y. Misuoka, S. Yamamoto, and H. Hoshi, "Optical pattern recognition with LAPS-SLM (2), feedback joint transform correlator using LAPS-SLM", Proceedings of the SPIE Vol. 1211, pp. 284-295 (1990)

[25] M.S. Alam and M.A. Karim, "Improved correlation discrimination in a multi-object bipolar joint transform correlator", Optics \& Laser Technology 24, 45-50 (1992).

[26] M. S. Alam and M. A. Karim, "Enhanced correlation discrimination using binary joint transform correlation with feedback", Microwave and Optical Technology Letters 5, pp. $752-757$ (1992).
[27] F. T. S. Yu and F. Cheng, "Multireference detection using iterative joint transform operation with composite filtering", Microwave and Optical Technology Letters 7, pp. 653-658 (1994).

[28] J. Edwards and H. Murase, "Appearance matching of occluded objects using coarse-to-fine adaptive masks", 1997 IEEE Computer Society Conference on Computer Vision and Pattern Recognition (Cat. No.97CB36082), pp. 533-539 (1997).

[29] N. Kasama, Y. Mitsuoka, T. Iwaki, and S. Yamamoto, "Effects of nonlinear parameters in feedback joint transform correlator (FJTC) (1)", Proceedings of the SPIE Vol. 1772, pp. 51-62 (1992).

[30] R. Chellappa, C. L. Wilson, and S. Sirohey, " Human and machine recognition of faces: a survey", Proceedings of the IEEE 83, pp. 705-740 (1995).

[31] B. Javidi, J. Li, and Q. Tang: "Optical implementation of neural networks for face recognition by the use of nonlinear joint transform correlators", Applied Optics 34 , pp. 3950-3962 (1995).

[32] D. Casasent, "An optical correlator feature extractor neural net system", Optical Engineering 31, pp. 971-978 (1992).

[33] D. Casasent, "General-purpose optical pattern recognition image processors", Proceedings of the IEEE 82, pp. 1724-1734 (1994)

[34] D. A. Jared, K. M. Johnson, and G. Moddel, "Joint transform correlation using an amorphous silicon ferroelectric liquid crystal spatial light modulator", Proceedings of the SPIE Vol. 1151, pp. 148-154 (1989).

[35] B. Javidi, G. Zhang, A. H. Fazlollahi, and U. Efron, "Application of a wire-grid-mirror liquid-crystal light valve in a nonlinear joint transform correlator", Applied Optics 33, pp. 2834-2841 (1994).

[36] T. Grycewicz, "Fourier-plane windowing in the binary joint transform correlator for multiple target detection", Applied Optics 34 , pp. 39333941 (1995).

[37] B. Javidi, "Nonlinear joint power spectrum based optical correlation", Applied Optics 28 , 2358-2367 (1989).

[38] B. Javidi and D. Painchaud, "Distortion-invariant pattern recognition with Fourier-plane nonlinear filters", Applied Optics 35 , pp. 318-331 (1996).

[39] D. I. Barnea and H. F. Silverman, "A class of algorithms for fast digital image registration", IEEE Transactions on Computers C-21, pp. 179-186 (1972).

[40] D. Casasent and D. Psaltis, "Scale invariant optical correlation using Mellin transform", Optics Communications 17, pp. 59-63 (1976).

[41] F. T. S. Yu, X. Li, and E. Tam, "Detection of rotational and scale varying objects with a programmable joint transform correlator", Proceedings of the SPIE Vol. 1053, pp. 167-176 (1989).

[42] K. Chalasinska-Macukow and J. Piliszek, "Scale-invariant limitations of anamorphic Fourier correlators", Optics Communications 74, pp. 25-30 (1989).

[43] B. S. Reddy and B. N. Chatterji, "An FFT-based technique for translation, rotation, and scale-invariant image registration", IEEE Transactions on Image Processing IP-5, pp. 1266-1271 (1996).

[44] J. Altmann and H. J. P. Reitböck, "A fast correlation method for scale- and translation-invariant pattern recognition", IEEE Transactions on Pattern Analysis and Machine Intelligence, PAMI-6, pp. 46-57 (1984).

[45] O. Perez and M. A. Karim: "Joint transform correlator performance enhancement by a logarithmic-polar transformation", Optical Engineering, 32, pp. 2726-2734 (1993). 